\title{
MAGNETIC CIRCULAR DICHROISM STUDIES OF HEMOGLOBIN
}

\section{THE' REEUCTION OF FERRIHEMOGLOBIN BY FERROCYTOCHROME $b_{5}$ AND CHARACTERIZATION OF THE HIGH-SPIN HYDROXY SPECIES OF MIXED-VALENCE HEMOGLOBIN}

\author{
David A. JUCKETT * and Donald E. HULTQUIST \\ Department of Biological Chemisty. University of Michigan School of Medicine, Ann Arbor, MI, U.S.A.
}

Received Ist November 1983

Accepted 4th January 1984

Key words: Magnetic circular dichroism; Methemoglobin reduction; Ferrihemoglobin; Hemoglobin; Hydroxymethemoglobin: Cylochrnme $b_{s}$

\begin{abstract}
The final step in the erythrocyte methemoglobin reduction pathway, the transfer of an electron from cytochrome $b_{s}$ io methemoglobin, has been studied using magnetic circular dichroism spectroscopy. Spectral analysis allowed us to determine accurately the concentration of each redox species in mixtures of the two heme-proteins and to follow simultaneously the kinetics of the appearance or disappearance of each of these species during reduction reactions. Our analysis detected a substantial increase in the high-spin hydroxymethemoglobin species in the partially reduced bovine hemoglobin tetramer. This species was sensitive to the degree of reduction and $\mathrm{pH}$, and was spectrally similar to fluoride methemoglobin. At $\mathrm{pH} 7.8$. 100\% of the hydroxide component of methemoglobin was in the high-spin form when two or more subunits were in the ferrous form. Kinetic analysis of bovine methemoglobin reduction yielded values for the apparent first-order rates for the tetrameric species possessing four, three, two, and one ferric subunit. Further analysis showed that the reduction kinetics can also be described by an equilibrium state, pure competitive inhibition model for enzyme catalysis in which ferrous and ferric subunits of hemoglobin compete for cytochrome $b_{5}$. This analysis generated a $K_{\mathrm{D}}$ that depends on icnic strength and hemoglobin tetramer conformation, a $V_{\max }$ that was independent of these factors, and an inhibition constant that was equal to $K_{\mathrm{D}}$. This model is consistent with the hypothesis that the reduction of methemoglobin can be separated into two steps, the ionic interaction between cytochrome $b_{5}$ and hemoglobin and the electron transfer.
\end{abstract}

\section{Introduction}

Magnetic circular dichroism (MCD) spectroscopy has been used to study a number of hemeproteins, including cytochrome $b_{5}$ and hemoglobin [1-7]. The MCD spectra of these heme-proteins have been shown to be very sensitive to spin, oxidation, and ligand states of the heme. The visible region of the reduced cytochrome $b_{5}$ spec-

- Present address: Department of Biochemistry, St Jude Children's Research Hospital, 332 N. Lauderdale, P.O. Box 318, Memphis. TN 38101, U.S.A. trum contains a very sharp, intense A term [2] which allows small concentrations of this species to be detected in the presence of other heme-proteins [3]. Hemoglobin MCD spectra show unique differences with various ligands in both the oxidized and reduced states [4-7].

Evidence that cytochrome $h_{s}$ and methemoglobin form a complex has been provided by isoelectric focusing studies [8], by kinetic studies of the transfer of electrons from ferrocytochrome $b_{s}$ to methemoglobin [9], and by spectral perturbation in the visible region of the ferric forms of hemoglobin and solubilized liver microsomal cyto- 
chrome $b_{5}[10]$. The significance of such a complex lies with the observation that methemoglobin reduction by the enzymatic system of crythrocytes proceeds through such a complex and that the rate of methemoglobin reduction under physiological conditions appears to be dependent on the concentration of the complex [9].

In this study we have further examined the interaction of reduced cytochrome $b_{5}$ with hemoglobin and described the results in terms of two models. A nonreversible first-order model fits the data and yields values of the rate constants for tetrameric hemoglobin species posscssing four, three, two and one ferric subunit. A MichaelisMenten equilibrium state model describes the data well in terms of formation of a cytochrome $b_{5}$-ferric hemoglobin complex, electron transfer between hemes of this complex, and competition between ferrous and ferric hemoglobin subunits for the cytochrome. During the reduction to deoxyhemoglobin, we observed in mixed-valence hemoglobin the conversion of hydroxymethemoglobin subunits to a pure high-spin species with an MCD spectrum similar to that of fluoromethemoglobin.

\section{Materials and methods}

\subsection{Materials}

NAD, NADH, Bis-Tris, and Trizma base were obtained from Sigma Chemical Co. Horse heart inyoglobin was obtained from ICN Biochemicals. Bovine liver microsomal cytochrome $b_{5}$ reductase was obidined by the method outlined previously [11] and was solubilized with chymotrypsin by the method of Spatz and Strittmatter [12]. Bovine liver microsomal cytochrome $b_{5}$ was purified by the method of Ozols [13]. The cytochrome $b_{5}$ was incubated with bovine liver lysosomal cathepsin $D$ and the resulting solubilized cytochrome was purified to near-homogeneity by chromatographic procedures (Schafer, Peters and Yultquist, unpublished data). Bovine hemoglobin was isolated from fresh blood (purebred Hereford) by the meihod of Antonini and Brunori [14] and was further purified on DE-52 cellulose. The hemoglobin was bound to the column at $\mathrm{pH} 8.6$ in a Tris- $\mathrm{Cl}$ buffer of ionic strength $0.02 \mathrm{M}$. Bovine hemoglobin A was then eluted with a decreasing $\mathrm{pH}$ buffer at constant ionic strength.

Hemoglobin was oxidized with a 3-4-fold excess of potassium ferricyanide in a buffered solution. The solution was then passed through Amberlite MB-3 (Sigma) mixed-bed ion-exchange resin to remove all ions. Methemoglobin was stored at $4^{\circ} \mathrm{C}$ at a concentration of about $2 \mathrm{mM}$ and was used within 5 days. All methemoglobin and hemoglobin concentrations are expressed on a per heme basis.

\subsection{Apparatus and data collection}

MCD spectra were taken on a Jasco J-40C spectropolarimeter equipped with an electromagnet capable of generating a field strength of 15000 G. The spectropolarimeter was interfaced to a Nova III computer and a Tracor $1500 \mathrm{~N}$ signal analyzer. Spectra acquisition, storage and baseline subtraction were facilitated by the computer/ signal analyzer pair. The spectra consisted of 1024 data points which were fed out to an Ohio Scientific Challenger II microcomputer which then stored the spectra on floppy disks. Each scan was condensed to 128 points, for subsequent multiple linear regression analysis, by averaging eight adjacent points sequentially along the spectrum. All spectral analysis and plotting was performed on the Challenger II using basic programs and an ADM 3a terminal retrofitted with the RetroGraphics plotting hardware.

Spectra were obtained from the spectropolarimeter at $50 \mathrm{~nm} / \mathrm{min}$, with the sensitivity scale set at 20 mdegree $/ \mathrm{cm}$, a 1.0 time constant, $1 \mathrm{~nm}$ spectral bandwidth, $15000 \mathrm{G}$ magnetic field, and the sample temperature of $4-8^{\circ} \mathrm{C}$. Baselines were scanned four to six times, averaged, and then stored in the Nova/Tracor. Anaerobic cuvettes with path lengths of $1 \mathrm{~cm}$ and $2 \mathrm{~mm}$ were used. A single spectrum containing MCD plus $C D$ was obtained at each time point during an enzymatic reduction. The natural $C D$ in the $670-470 \mathrm{~nm}$ range was very small for hemoglobin, myoglobin, and cytochrome $b_{\mathrm{s}}$ and did not interfere with the analyses. 


\subsection{Methemoglobin reduction}

Methemoglobin was reduced by the electrontransport chain consisting of NADH, proteasesolubilized microsomal cytochrome $b_{5}$ reductase and protease-solubilized microsomal cytochrome

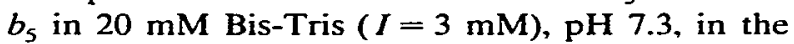
absence and presence of $\mathrm{NaCl}$ and inositol hexaphosphate (IHP). We used concentrations of components that maintained cytochrome $b_{5}$ primarily in a reduced form in the presence of methemoglobin. This allowed us to examine the transfer of electrons from cytochrome $b_{5}$ to hemoglobin with the proteins in the physiologically relevant redox states. Hemoglobin concentrations were maintained at $50 \mu \mathrm{M}$ or greater to assure a minimum of $90 \%$ of the hemoglobin in the tetramer form at the highest ionic strength used. We used the value of $4 \times 10^{6} \mathrm{M}^{-1}$ for the dimer-tetramer association constant of bovine hemoglobin [15] (derived for an ionic strength of $0.225 \mathrm{M}$ ) to arrive at this lower limit.

Under aerobic conditions, NADH was added to a mixture of the proteins to start the reaction. Under anaerobic conditions, hemoglobin was placed in the sidearm of a Thunberg cuvette and the remaining components placed in the main body of the cuvette. The cuvette was then repeatedly evacuated and flushed with $\mathrm{N}_{2}$ which had been purified through an activated Ridox (Fisher Scientific) column and then bubbled through distilled water. Any residual $\mathrm{O}_{2}$ was scrubbed from the system by the oxidation of ferrous cytochrome $b_{5}$. After approx. $1 \mathrm{~h}$ the methemoglobin was tipped in and its recuction was followed by MCD at $8^{\circ} \mathrm{C}$. MCD spectra (duration of $\operatorname{scan}=4 \mathrm{~min}$ ) were taken at 5-min intervals after the initiation of the enzymatic reductions and were continued until most of the methemoglobin was reduced.

\subsection{Data analysis}

The relative contributions of the 'pure' oxidation states of the heme-proteins present in a sample spectrum were determined using multiple linear regression analysis. The spectra of the pure states were used as the independent variable $(X,(j))$ in the equation:

$$
Y(j)=a_{0}+a_{1} X_{1}(j)+a_{2} X_{2}(j)+\ldots+a_{n} X_{n}(j)
$$

where $j$ represents the number of data points in each spectrum and therefore the number of data sets $\left(Y, X_{1}, X_{2}, \ldots X_{n}\right)$ used in the regression analysis.

Due to a slight drifting in the monochrometer, the MCD signals were shifted as a function of time. It was necessary to analyze for this shift because the standard spectra $\left(X_{t}\right)$ and sample spectra were taken at times which differed by hours or days. Since the shifts were small ( $<2 \mathrm{~nm}$ ) we obtained a first-order approximation of the shift of a standard spectrum by including its first derivative in eq. 1 as an additional independent variable. This led to the equation:

$$
\begin{aligned}
Y= & a_{0}+a_{1} X_{1}+a_{2} X_{2}+\ldots+b_{1} \frac{\mathrm{d} X_{1}}{\mathrm{~d} \lambda} \\
& +b_{2} \frac{\mathrm{d} X_{2}}{\mathrm{~d} \lambda}+\ldots
\end{aligned}
$$

The coefficient $b_{i}$ was divided b; $a_{i}$ to determine the shift, $\Delta \lambda_{f}$; the shift is determined from the coefficient of the first dcrivative of the contribution of $X_{\text {, }}$ to the sample spectrum, which is $\Delta \lambda, \mathrm{d}\left(a, X_{1}\right) / \mathrm{d} \lambda$. Since this term is equivalent to $\Delta \lambda_{i} a_{1} \mathrm{~d} X_{1} / \mathrm{d} \lambda_{,} b_{1}=\Delta \lambda_{1} a_{1}$, and $\Delta \lambda_{1}=b_{1} / a_{1}$. Analysis for shifts was routinely included only on the standard spectra of reduced cytochrome $b_{5}$ and oxyhemoglobin because of the narrow, intense bands in these spectra.

It was occasionally necessary to reduce the number of independent variables in eq. 1 . Since the oxidized and reduced forms of a single protein species are related to the total amount of that protein by the equation $c=a_{1}+a_{2}$, the value of $c-a_{1}$ can be substituted for $a_{2}$ in eq. 1 or 2 where the subscripts 1 and 2 represent oxidized and reduced species of a single protein, respectively. This substitution yields an equation with one less independent variable and one less degree of freedom. The equation to be solved is then:

$Y-c X_{2}=a_{0}+a_{1}\left(X_{1}-X_{2}\right)+a_{3} X_{3}+\ldots$

(This general form can be expanded for use when more than one pair needs to be set equal to a 
constant.) The use of this equation can be advantageous when the spectrum of one of the protein species is of low intensity, or when it resembles the spectral characteristics of a species from another protein. If a low-intensity species has a coniplementary redox state with a highly intense, highly unique signal, then use of eq. 3 couples the evaluation of the coefficients $a_{1}$ and $a_{2}$ to the highly unique species and thereby gives a more accurate determination of the less unique signal. Even with hemoglobin present in a 10 -fold excess, the weak signal of oxidized cytochrome $b_{5}$ was successfully determined by coupling it to the highly intense reduced cytochrome $b_{\mathrm{s}}$ signal.

The computer algorithm for the multiple regression analysis was adapted from that of Bevington [16]. Incorporation of the first derivatives and the coliplea solutions originated with the authors.

2.5. Kinetic analysis model I: $A$ sequential, first-order approach

A simple, first-order model was used to predict the reduction of each subunit of the methemoglohin tetramer. This model was based on the scheme:

$\mathrm{H}_{4} \stackrel{k_{3}}{\rightarrow} H_{3} \stackrel{k_{3}}{\rightarrow} H_{2} \stackrel{h_{2}}{\rightarrow} H_{1} \stackrel{k_{1}}{\rightarrow} H_{0}$

where the $H$, represent the concentrations of tetramer with $i$ subunits in the ferric state. Since each step is first-order it follows, therefore, that

$\mathrm{d} H,(t) / \mathrm{d} t=-k, H,(t)$

At time zero, all the hemoglobin is in the ferric form (i.e., $H_{3}, H_{2}, H_{1}$ and $H_{0}$ equal zero). The amount of total ferric heme as a function of time is represented by

$\mathrm{Fe}^{3+}(t)=\sum_{t} i H_{t}(t), \quad i=4,3,2.1$

The concentration of each tetrameric species can be numerically estimated by the following set of equations:

$$
\begin{aligned}
& H_{4}(t+\Delta t)=H_{4}(t)+\Delta H_{4}(t) \\
& H_{i}(t+\Delta t)=H_{2}(t)+\Delta H_{2}(t)-\Delta H_{t+1}(t) \\
& \quad i=3,2,1
\end{aligned}
$$

and
$H_{0}(t+\Delta t)=H_{0}(t)-\Delta H_{1}(t)$

where

$\Delta H_{i}(t)=-k_{i} H_{1}(t) \Delta t$,

Eqs. 5 and 6 were used to generate the smooth curves in fig. 3 after estimates of $k_{i}$ were obtained.

This reaction scheme can also be integrated into an exact analytical form [17] that directly gives each $H_{r}$ as a function of time. The integrated rate equations were used in an iterative least-squares method described by Olson [17] to arrive at estimates of $k$, using the experimentally measured data, $\mathrm{Fe}^{3+}(t)$. The estimates of $k$, were obtained by minimizing a computed error function containing $k_{i}+\Delta k_{1}$ and then solving for $\Delta k_{i}$. This procedure was performed iteratively until the $k$, terms converged to stable values. This method required initial guesses of $k_{i}$ and they were often the critical factor determining convergence. It was also apparent that local minima existed in the 'solution space' for the four $k_{i}$ terms that resulted in convergence to slightly different values depending on the initial guesses. We, therefore, cannot be sure that the estimates of $k_{i}$ given here represent the true global minimum for each data set but they are adequate estimates because in all cases the differences between the four $k$, terms of a solution were very much greater thar. the slightly different values obtained for each $k$, with various initial guesses.

\subsection{Kinetic analysis model II: An equilibrium kinet- ics, competitive inhibition approach}

An equilibrium state, pure competitive inhibition formulation was also used to describe the kinetic data for reduction of methemoglobin. This model assumes that cytochrome $b_{5}$ takes the role of enzyme, methemoglobin is the substrate, and ferrous hemoglobin is a pure competitive inhibitor. The velocity equation for an increase in the total ferrous heme, $Q$, can be constructed as the sum of velocity components for each valence form of the tetramer and is given by:

$\frac{\mathrm{d} Q}{\mathrm{~d} t}=\frac{V_{\max }}{4}\left\{\frac{4 H_{4}}{K_{\mathrm{D}}+\frac{K_{\mathrm{D}}}{K_{\mathrm{I}}} Q+H_{4}}+\frac{3 H_{3}}{K_{\mathrm{D}}+\frac{K_{\mathrm{D}}}{K_{\mathrm{I}}} Q+H_{3}}\right.$ 


$$
\left.+\frac{2 H_{2}}{K_{\mathrm{D}}+\frac{K_{\mathrm{D}}}{K_{1}} Q+H_{2}}+\frac{H_{2}}{K_{\mathrm{D}}+\frac{K_{\mathrm{D}}}{K_{1}} Q+H_{1}}\right\}
$$

where $V_{\max }$ is the maximum velocity for reaction of cytochrome $b_{s}$ with a fully oxidized hemoglobin tetramer, $H_{i}$ the concentrations of hemoglobin tetramer with $i$ oxidized subunits, $K_{3}$ the inhibition constant arising from the nonproductive complexation of ferrous cytochrome $b_{\mathrm{s}}$ with a ferrous hemoglobin subunit, and $K_{\mathrm{D}}$ the dissociation constant characterizing the complexation of ferrous cytochrome $b_{s}$ with a methemoglobin subunit. The degree of inhibition by ferrous subunits increases with increasing [Q] which is a common factor in all terms while substrate concentration $H_{j}$ for each term depends on the instantaneous amount of that tetrameric form. The factors $4,3,2$ and 1 are incorporated because of the reactivity cross-section of the various tetramer forms, thus, eq. 7 contains three unknown parameters, $V_{\max }, K_{D}$ and $K_{1}$, each with an intrinsic meaning within the framework of the model.

This model was derived using Michaelis-Menten kinetics under equilibrium conditions as applied to the following reaction scheme:

$H_{1}+b_{5}^{2+} \stackrel{k_{i}^{\prime}}{\underset{k_{i}^{\prime}}{\Rightarrow}} H_{1} b_{5}^{2+} \stackrel{k_{i}^{\prime \prime}}{\rightarrow} H_{i-1}+b_{5}^{3+}, \quad i=4,3,2,1$

where $b_{5}^{2+}$ and $b_{5}^{3+}$ represent the ferrous and ferric forms of the cytochrome. (Under the conditions of this work, the concentration of $b_{5}^{3+}$ is negligible.) Eq. 7 was derived from this reaction scheme under the assumption that each rate constant in eq. 8 is the same for all values of $i$ and that equilibrium conditions are appropriate. The latter assumption requires that $k^{\prime \prime} \gg k^{\prime \prime \prime}$ and therefore yields a $K_{\mathrm{D}}$ instead of a $K_{M}$ as in the typical steady-state kinetic expression. (Using the $V_{\max }$ and $K_{D}$ values derived in this work and a very conservative lower limit estimate of $k^{\prime}=1 \times 10^{6} \mathrm{M}^{-1} \mathrm{~s}^{-1}, k^{\prime \prime}$ is greater than $k$ "' by at least 2 orders of magnitude for all the conditions studied.) The validity of these two assumptions was examined by trying to fit eq. 7 to the data using $\Delta Q / \Delta t$ and values of $H_{i}$ generated by eqs. 5 and 6 obtained with the best-fit values of $k_{r}$. Since the kinetic curves generated by using $k$, (section 2.5) excellently reproduced the actidal data, they served as an ideal way to obtain approximations to $H_{\text {, }}$ and to obtain more accurately $\Delta Q / \Delta t$ by making $\Delta t$ smaller than the time interval between actual data points. The value of $V_{\max }$ was obtained from the Lineweaver-Burk plot (fig. 4) and $K_{1}$ was set equal to $K_{\mathrm{D}}$, representing the most simple case for the interaction of the two proteins *. With $V_{\max }$ fixed and $H_{i}$ and $\Delta Q / \Delta t$ generated for each time point, the test of cq. 7 simplified to the ability of a single $K_{D}$ vaiue to satisfy the equation at all points in the reduction: A $K_{\mathrm{D}}$ value can be generated for each time point by solving eq. 7 for $K_{\mathrm{D}}$. This was done by rewriting eq. 7 as a fourth-order polynomial in $K_{\mathrm{D}}$ and determining the four roots at each time point. In all cases examined, only one of the four roots was positive and that was taken as the physically relevant solution. The roots obtained are shown in fig. 5 and described in sectivar 3.2 .

\subsection{Elucidation of high-spin hydroxymethemoglobi:?}

Spectra taken during the course of reductions were initially analyzed assuming that hemoglobin was present only as deoxyhemoglobin and methernoglobin, as represented by the spectra of pure deoxyhemoglobin and pure methemoglobin at the pH of the reduction. This type of analysis yielded values for the contributions of these two hemoglobin species at each point in the reduction. The sum of methemoglobin and deoxyhemoglobin at each point did not equal the total hemoglobin present, however, and the residual spectrum (the sample spectrum, $Y$, minus the computed contributions of known components) was not flat. These observations indicated that at least one other unique species was present in the MCD spectrum during reduction. The maximum deviation from a

\footnotetext{
* The inhibition constant. $K_{1}$, incorporated in eq. 7 represents a dissociation constant for the nonproductive complexation of ferrous cytochrome $b_{5}$ with a ferrous hernoglobin subunit. Since eq. 7 was derived using equilibrium rather than steady-state kinetics, it contains $K_{\mathrm{D}}$ which is the dissociation constant for the productive complexation of the two proteins. Both $K_{t}$ and $K_{\mathrm{D}}$ are equilibrium constants and therefore it is reasonable to test the case where $K_{I}=K_{D}$.
} 
best fit resulting from the unknown species occurice midway through the reduction under anaerobic conditions.

The spectrum of the unknown species was determined by manipulation of the contributions of met and de:xy forms of herroglobin in such a way that the following equations were satisfied at each time point in the reduction:

$$
\begin{aligned}
& a_{1} \epsilon_{\text {met }}(\lambda)+a_{2} \epsilon_{\text {deary }}(\lambda)+a_{3} \epsilon_{\text {unh }}(\lambda)=A_{\text {oh }}(\lambda) \\
& a_{1}+a_{2}+a_{3}=[\mathrm{Hb}]_{\text {total }}
\end{aligned}
$$

where the $\epsilon(\lambda)$ terms represent the MCD spectra (in units of $[\theta]_{M}$ ) for each species and $A_{\mathrm{oh}}$ is the observed MCD spectrum at each time point. Since $a_{1}, a_{2}, a_{3}$ and $\epsilon_{\text {unk }}(\lambda)$ were not known, an iterative approach was used by varying $a_{1}$ and $a_{2}$ and using the residual spectrum from the previous iteration as $\epsilon_{\text {unk }}(\lambda)$. This proceeded until the $\epsilon_{\text {unk }}(\lambda)$ obtained plus $\epsilon_{\text {met }}(\lambda)$ and $\epsilon_{\text {deoxy }}(\lambda)$ could be used to fit all the time points of the reduction while simultaneously satisfying eq. 10.

Since the unknown spectrum resembled highspin fluoromethemoglobin, the latter spectrum was routinely used in all spectral analyses reported here.

\section{Results}

\subsection{MCD spectra of hemoglobin and cytochrome $h_{5}$}

The MCD spectra of various ligand and valence states of bovine cytochrome $b_{5}$ and hen.oglobin a:e stown in fig. 1 for the wavelength regions $370-470$ and $470-670 \mathrm{~nm}$. The spectra illustrate the sensitivity of MCD to ligand and spin state of the heme-proteins. For all of the protein states shown, the visible region has more characteristic band features than the Soret region. The spectra in the visible region were used. therefore, in the multiple regression analysis to determine relative oxidation state contributions of hemoglobin and cytochrome $b_{5}$ during reduction.

\subsection{Methemoglobin reduction}

Reduction of methemoglobin was carried out under conditions which kept the cytochrome $b_{s}$ in

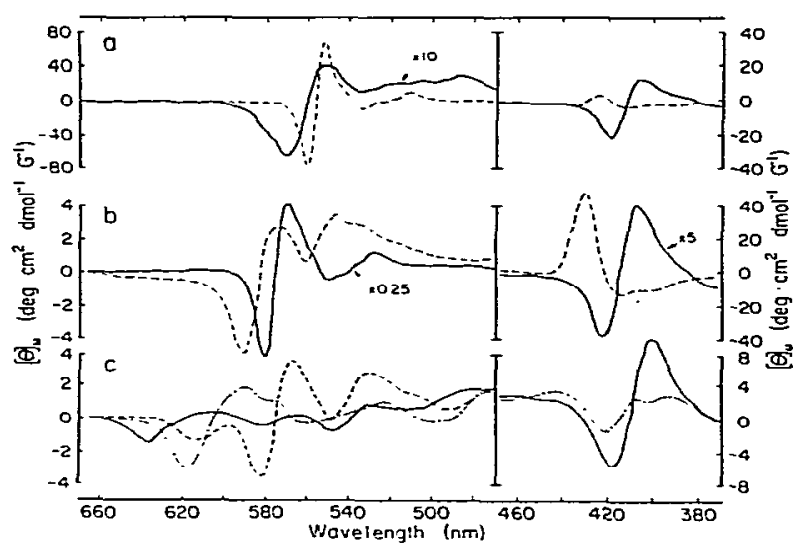

Fig. 1. MCD spectra of various forms of hemoglobin and cytochrome $b_{s}$. Final concentrations: $10 \mu \mathrm{M}$ cytochrome $b_{S}$ and $40 \mu \mathrm{M}$ hemoglobin in $20 \mathrm{mM}$ Bis-Tris ( $\mathrm{pH} \mathrm{7.3)}$ at $8^{\circ} \mathrm{C}$. Ferrous species were obtained by addition of dithionite. Methemoglobin was prepared as described in section 2.1. Top: reduced cytochrome $b_{s}\left({ }_{-}-\right)_{\text {, oxidized cytochrome } b_{s}}$ (-). Middle: oxyhemoglobin (- $(-$ ), deoxyhemoglobin $(-\ldots)$. Bottom: aquomethemoglobin (methemoglobin prepared in the presence of $100 \mathrm{mM} \mathrm{NaF}$ $(\cdot-\cdot-)$. mixed-spin hydroxide methemoglobin at $\mathrm{pH} 10$ $(--\infty)$.

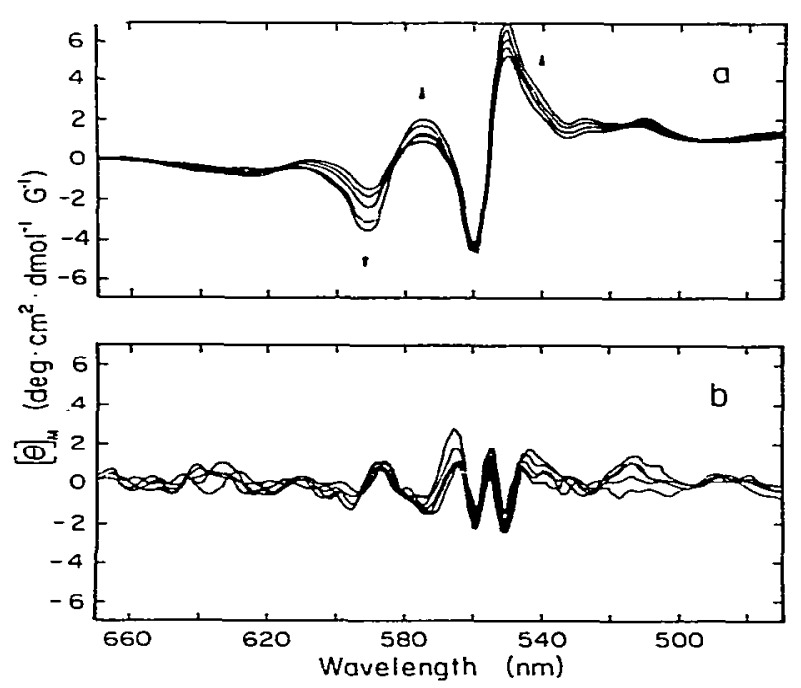

Fig. 2. MCD spectral analysis of methemoglobin reduction. (a) Spectra at selected times during reduction in the absence of oxygen. Arrows indicate spectral changes with time. Specific data are listed in table 1. (b) Residual spectra at the same time points computed after best-fit analysis by linear regression. 
Table 1

Results of the multiple regression analysis of reduction of methemoglobin and ferricytochrome $b_{5}$ under anaerobic conditions

Reaction conditions: $8^{\circ} \mathrm{C}$. pH 7.4; final concentrations: $0.1 \mathrm{M} \mathrm{NaCl}, 7 \mathrm{mM}$ Bis-Tris buffer. $1 \mathrm{mM} \mathrm{NADH}$, and 0.05 unit cytochrome $b_{\mathrm{s}}$ reductase $/ \mathrm{ml}$.

\begin{tabular}{|c|c|c|c|c|c|c|c|c|}
\hline \multirow[t]{2}{*}{ Tine (min) } & \multicolumn{6}{|c|}{ Concentrations $(\mu \mathrm{M})$} & \multirow{2}{*}{$\begin{array}{l}\text { Shift (nm) } \\
\text { of cytochrome } b_{s}\end{array}$} & \multirow{2}{*}{$\frac{\mathrm{F}^{-}-\mathrm{Fe}^{3+} \mathrm{Hb}}{\text { Total } \mathrm{Fe}^{3+} \mathrm{Hb}} \times 100(\mathscr{G})$} \\
\hline & $\mathrm{H}_{2} \mathrm{O}-\mathrm{Fe}^{3+} \mathrm{Hb}$ & $\mathrm{F}^{-}-\mathrm{Fe}^{3+} \mathrm{Hb}$ & $\mathrm{Fe}^{2+} \mathrm{Hb}$ & $\mathrm{O}_{2}-\mathrm{Fe}^{2+} \mathrm{Hb}$ & Total Hb & $\mathrm{Fe}^{2+b_{s}}$ & & \\
\hline 2 & 36.1 & 1.9 & 6.7 & 0.66 & 45.4 & 4.41 & -0.17 & 5 \\
\hline 7 & 27.4 & 4.8 & 13.2 & 0.42 & 45.8 & 4.68 & -0.12 & 15 \\
\hline 12 & 23.0 & 5.4 & 17.0 & 0.44 & 45.8 & 4.83 & -0.15 & 19 \\
\hline 17 & 20.6 & 5.8 & 19.4 & 0.47 & 46.3 & 4.87 & -0.16 & 22 \\
\hline 32 & 15.8 & 5.4 & 24.1 & 0.55 & 45.8 & 4.97 & -0.02 & 25 \\
\hline 52 & 13.8 & 4.2 & 28.0 & 0.57 & 46.6 & 5.01 & -0.31 & 23 \\
\hline 97 & 9.5 & 3.3 & 33.2 & 0.87 & 46.9 & 4.99 & -0.19 & 26 \\
\hline 147 & 6.7 & 1.6 & 36.2 & 1.20 & 45.7 & 4.96 & -0.19 & 19 \\
\hline
\end{tabular}

virtually a completely reduced state as demonstrated in fig. $2 a$. The intensity of the signal of the reduced cytochrome $b_{5}$ (near $555 \mathrm{~nm}$ ) remained approximately constant as methemoglobin underwent complete reduction to deoxyhemoglobin. Maintenance of cytochrome $b_{\mathrm{s}}$ in the ferrous form was crucial to the study of the final step in the electron transfer to methemoglobin and the kinetics described in this section were interpretable only if this condition was strictly met. The complete analysis of the reduction of fig. $2 a$ is given in table 1. Using fluoromethemoglobin as an analog of the high-spin hydroxymethemoglobin species (see sections 2.7 and 3.3), a self-consistent analysis resulted, with total hemoglobin constant to within $1.1 \%$ standard deviation. The correction for the shift of the reduced cytochrome $b_{5}$ spectrum wa: small but necessary for a good fit. The residual
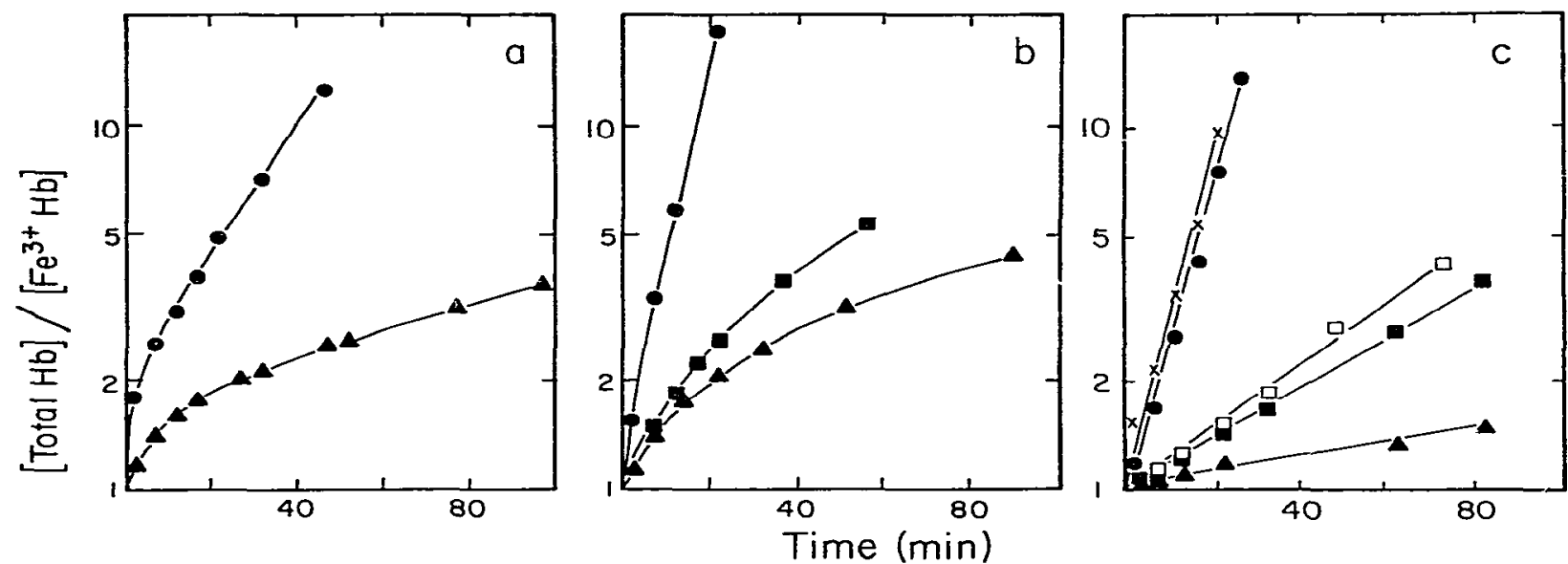

Fig. 3. Reduction of methemoglobin and metmyoglobin. The course of reaction is plotted such that slope is directly proportional to rate. Final concentrations: 0.01 unit of cytochrome $b_{5}$ reductase/ml. $1 \mathrm{mM} \mathrm{NADH,} \mathrm{and} 20 \mathrm{~m} . \mathrm{M}$ Bis-Tris (pH 7.3). (a) Anaerobic reduction of methemoglobin. Final concentrations: $50 \mu \mathrm{M}$ methemoglobin and $5 \mu \mathrm{M}$ cytochrome $b_{5}$. No addition $(\bullet-\longrightarrow), 0.1 \mathrm{M}$ $\mathrm{NaCl}(\Delta-\wedge)$ (b) Aerobic reduction of methemoglobin. Final concentrations; $50 \mu \mathrm{M}$ methemoglobin and $5 \mu \mathrm{M}$ cytochrome $b_{5}$.

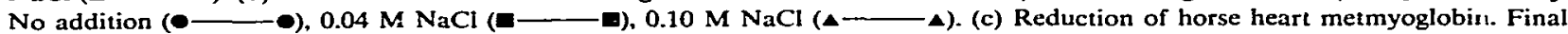
concentrations: $20 \mu \mathrm{M}$ metmyoglobin and $1 \mu \mathrm{M}$ cytochrome $b_{5}$. Anaerobic, no $\mathrm{NaCl}(\times \longrightarrow \times)$ anaerobic, $0.03 \mathrm{M} \mathrm{NaCl}$ $(\square-\square)$; aerobic, no $\mathrm{NaCl}(-\bullet)$; aerobic, $0.03 \mathrm{M} \mathrm{NaCl}(\square-\square)$; aerobic, $0.1 \mathrm{M} \mathrm{NaCl}(\llcorner-\square)$ 
spectra resulting from this analysis were essentially flat. except for some deflections in the $560 \mathrm{~nm}$ region which were due to a slight narrowing $(0.5-1.0 \mathrm{~nm})$ of the ferrocytochrome $b_{s}$ signal during reduction (fig. $2 b$ ). The use of $M C D$ and our analysis provided a suitable method to follow the various species involved in the reduction.

Time courses for the reduction of methemoglobin and metmyoglobin as a function of oxygen and ionic strength are sho:vn in fig. 3 . The data. plotted as $\ln$ ([total subunits]/[ferric subunits]) vs. time, would yield straight lines for those reactions that follow first-order kinetics. The plots of methemoglobin reduction do not yield straight lines but rather lines with slopes that decrease as a function of time (fig. $3 a$ and $b$ ). The slopes decrease with increasing ionic strength and there is a distinct biphasic nature to the anaerobic reduction. The plots for metmyoglobin, in contrast, appear to be first order with similar rates for aerobic and anaerobic conditions.

Analysis of the methemoglobin reduction kinetics using model 1 (as described in section 2.5) yielded four apparent first-order rate constants (designated $k_{4}, k_{3}, k_{2}$ and $k_{1}$ ) for the reduction of tetrameric hemoglobin possessing four, three, two. and one ferrir: subunit, respectively (table 2 ). These derived rate constants decreased in magnitude as the hernoglobin tetramer became more reduced. The smooth curves drawn through the data points in fig. $3 a$ and $b$ were generated from the derived rate constants $k$, using eqs. 5 and $6 a-d$. The derived curves show an excellent fit to the experimental data. The average deviation of these curves from the data points is approx. $0.2 \%$.

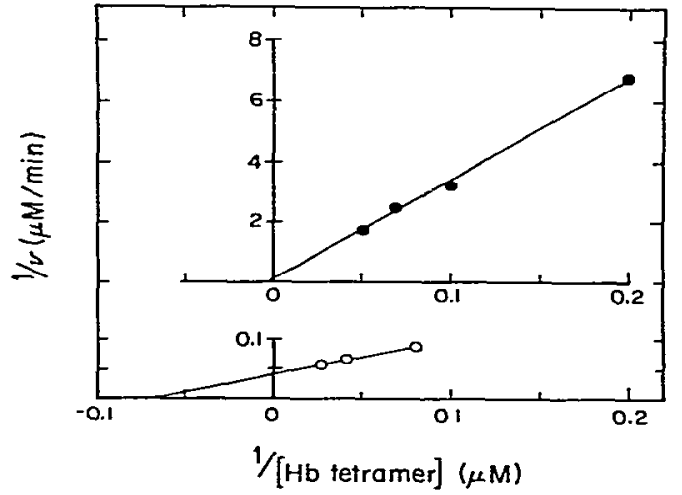

Fig. 4. Effect of methemoglobin concentration on the rates of electron transfer from ferrocytuchrome $b_{5}$ to hemoglobin with 100 or $10 \%$ of the subunits in the ferric form. Reduction was carried out in the abseice of oxygen with final concentrations of $1.0 \mathrm{mM}$ NADH, $20 \mathrm{mM}$ Bis-Tris buffer ( $\mathrm{pH} 7.3$ ), 0.05 unit cytochrome $b_{5}$ reductase $/ \mathrm{ml}$. $5 \mu \mathrm{M}$ cytochrome $b_{5}$. and total hemoglobin concentrations varying from 12.5 to $50 \mu \mathrm{M}$ hemoglobin tetramer. Lineweaver-Burk plots are shown for the initial velocities $(\mathrm{O}-\mathrm{O})$ and for the velocities at $90 \%$ reduction (-). At $90 \%$ completion, the calculation of concentration of tetramer containing one ferric subunit was based on the assumption that no more than one ferric subunit was present per tetramer.

The kinetics of reduction (fig. 3) were also analyzed using model II (as described in section 2.6) and values were obtained for $K_{\mathrm{D}}, K_{\mathrm{I}}$ and $V_{\max }$ which satisfied eq. 7 and reproduced the data. Values for $V_{\text {max }}$ were obtained from the Lineweaver-Burk plots (fig. 4) which represent velocities taken from the initial and final regions of reductions carried out with different total hemo-

Tathle 2

Apparent first-order rate constants for reduction of each valence species of tetrameric methemoglobin

For conditions and concentrations see fig. 3.

\begin{tabular}{|c|c|c|c|c|c|}
\hline \multirow[t]{2}{*}{$\mathrm{C}_{2}$} & \multirow[t]{2}{*}{ Ionic strength (M) } & \multicolumn{4}{|c|}{ Apparent rate constants $\left(\mathrm{min}^{-1}\right)$} \\
\hline & & $k_{4}$ & $k_{3}$ & $k_{2}$ & $k_{1}$ \\
\hline+ & 0.003 & 0.985 & 0.600 & 0.285 & 0.099 \\
\hline+ & 0.043 & 0.317 & 0.110 & 0.085 & 0.010 \\
\hline+ & 0.103 & 0.269 & 0.090 & 0.034 & 0.004 \\
\hline- & 0.003 & 1.25 & 0.800 & 0.092 & 0.039 \\
\hline- & 0.103 & 0.312 & 0.080 & 0.013 & 0.011 \\
\hline
\end{tabular}



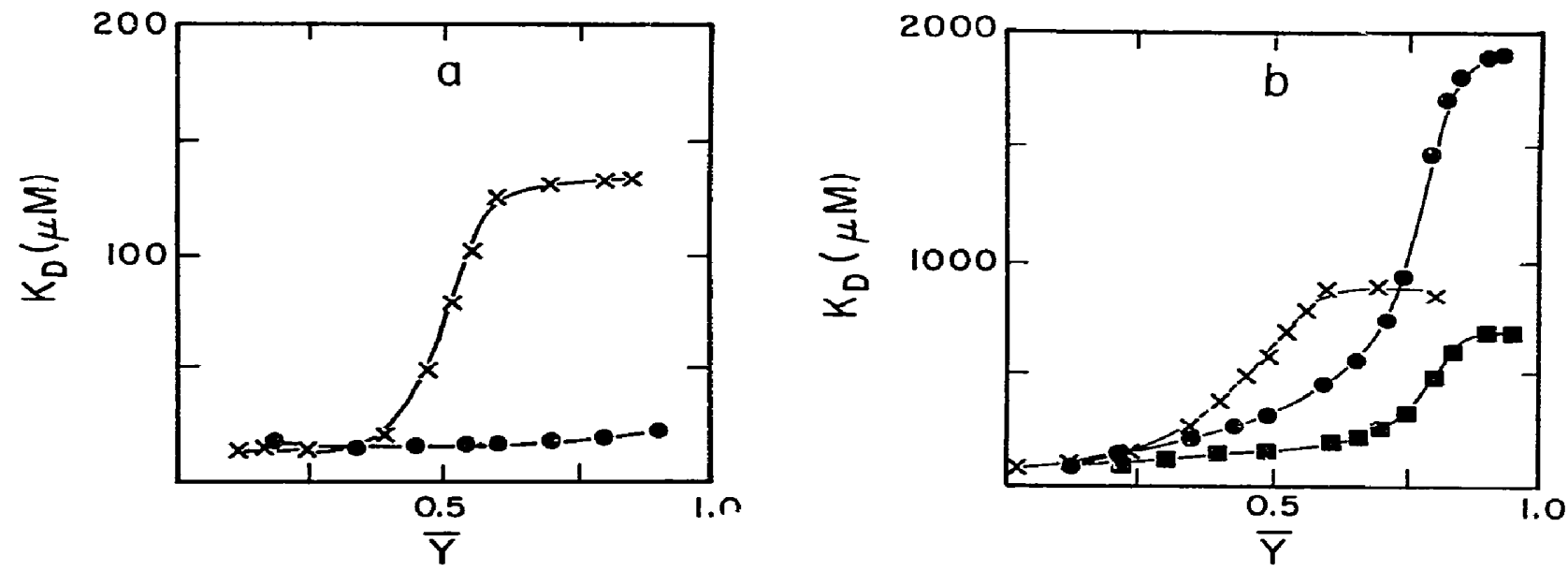

Fig. 5. Effect of degree of reduction, $\bar{Y}$, on $K_{\mathrm{D}} . K_{\mathrm{D}}$ were determined as described in section 2.6. (a) Low ionic strength (0.003 M). Aerobic conditions (- $-\infty)$, anaerobic conditions $(x-\infty)$. (b) Higher ionic strength. $\mu=0.1 M$. aerobic conditions $(\bullet-0.1 \mathrm{M}$, anaerobic conditions $(\times-\times) ; \mu=0.043 \mathrm{M}$, aerobic conditions (e- -0 ).

globin concentrations at low ionic strength in the absence of oxygen. These data are not sufficiently accurate to establish that $V_{\max }$ is constant but do suggest that $V_{\text {max }}$ is relatively stable while the apparent $K_{\mathrm{D}}$ values for the early and late stages of the reduction are vastly different. $V_{\text {max }}$ was, therefore, assumed to be constant and taken to be equal to $30 \mu \mathrm{M} / \mathrm{min}, K_{1}$ was set at $K_{\mathrm{D}}$, and the value of $K_{\mathrm{D}}$ that satisfied eq. 7 was determined at various theoretical time points in each reduction.

Plots of $K_{\mathrm{D}}$ vs. the fraction of reduced heme, $\bar{Y}$. are given in fig. 5. At low ionic strength $(I=0.003$ $M)$ and in the presence of oxygen, a relatively constant value of $K_{\mathrm{D}}$ was obtained throughout the reduction of methemoglobin (fig. 5a). This suggests a constant affinity between cytochrome $b_{5}$ and each tetrameric form of hemoglobin. In contrast, analysis of reductions in the absence of oxygen resulted in two values for $K_{\mathrm{D}}$, a low value early in the reduction and a high value late in the reduction. These two values are approximately an order of magnitude apart and are connected by intermediate values of $K_{\mathrm{D}}$ which define a fairly abrupt transition that occurs at the midpoint of the reduction. In the early part of the reduction the first two terms in eq. 7 predominate and, therefore, the lower value of $K_{\mathrm{D}}$ in this region characterizes the first two steps of the reduction. Similarly, the high values of $K_{\mathrm{D}}$ characterize the final two steps in the reduction. It follows, then, that the affinity of cytochrome $b_{5}$ for $H_{4}$ and $H_{3}$ is high while the affinity for $H_{2}$ and $H_{1}$ is low. Each intermediate value of $K_{\mathrm{D}}$ is not a meaningful value but is simply the best single estimate to the fourth-order polynomial representation of eq. 7 when significant amounts of $\mathrm{H}_{3}$ and $\mathrm{H}_{2}$ are present simultaneously. When reductions were carried out at higher ionic strength, all values of $K_{\mathrm{D}}$ were increased (fig. $5 \mathrm{~b}$ ) due to the diminished ionic affinity between the two proteins.

Reduction in the presence of oxygen at high ionic strength is more complicated. When hemoglobin is primarily in its ferric form, the $K_{\mathrm{D}}$ value is similar to the low $K_{\mathrm{D}}$ observed in the absence of oxygen. However, the $K_{\mathrm{D}}$ value steadily increases as $\bar{Y}$ increases. This is apparently due to the increasingly significant role of auto-oxidation of cytochrome $b_{5}$ as the overall rate of methemoglobin reduction decreases during the course of the reaction. The auto-oxidation is mathematically equivalent to an increased inhibition of the interaction of methemoglobin with cytochrome $b_{5}$ and is manifested as an increasing $K_{\mathrm{D}}$ in the calculation. 


\subsection{Detection of high-spin hydroxymethemoglobin}

Using the multiple regression analysis technique (sections 2.4 and 2.7), a previously unrecognized increase in high-spin hydroxymethemoglobin was discovered in partially reduced hemoglobin in the absence of oxygen. The MCD spectrum of this species is shown in fig. 6. The spectrum is distinct from those of aquomethemoglobin and mixed-spin hydroxymethemoglobin at $\mathrm{pH} 10$, but there is a striking similarity to the spectrum of fluoromethemoglobin. The presence of this species can be seen as a skewing of the $640 \mathrm{~nm}$ trough of the high-wavelength charge-transfer band which is normally due to aquomethemoglobin. An enlarged view of the high-wavelength region taken from the midpoint of the reduction in fig. $2 a$ is shown in fig. 7 (dotted line). There is clearly a negative deflection near $620 \mathrm{~nm}$ due to the contribution of a signal with a negative maximum at a lower wavelength than that of aquomethemoglobin and close to that of fluoromethemoglobin. Since this species shows charge-transfer bands in siruilar regions to those of fluoromethemoglobin it is very likely a high-spin form of methemoglobin. In contrast to methemoglobin reduction, metmyoglobin reduction proceeds with a simple monotonic decrease in the $640 \mathrm{~nm}$ trough with no skewing in the 610-630 nm region (data not shown).

Analysis of a reduction of methemoglobin at $\mathrm{pH} 7.8$ for mixed-spin hydroxymethemoglobin, aquomethemoglobin, and fluoromethemoglobin (fig. 8) led to tentative identification of the high-

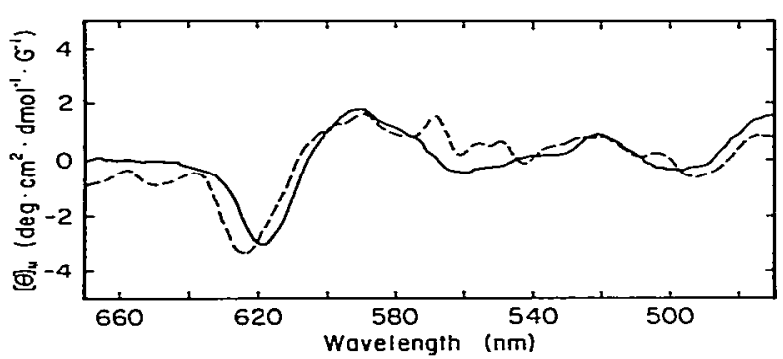

Fig. 6. Calculated MCD spectrum of the high-spin species of mixed-valence hemoglobin. Spectrum calculated as described in section 2.7 (-. - . -). spectrum of fluoromethemoglobin determined as described in fig. 1 ( $(-)$.

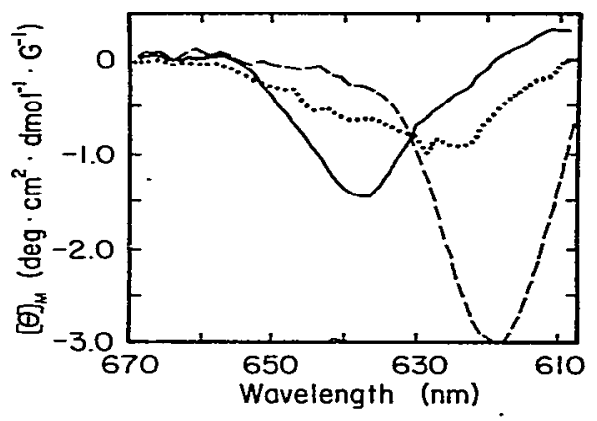

Fig. 7. MCD spectrum of partinlly reduced methemoglobin showing the contribution of the high-spin species. Methemoglobin was brought to approx. $50 \%$ reduction by incubation for $17 \mathrm{~min}$ (see fig. 2 and table 1). Low-energy region of the MCD visible spectrum of the partially reduced methemoglobin $(\cdots \cdots)$. aquomethemoglobin $(\longrightarrow)$ and fluoromethemoglobin (-- - - -).

spin component as high-spin hydroxymethemoglobin. As reduction proceeded, the mixed-spin hydroxide signal of methemoglobin decreased and the pure high-spin signal increased in a $1: 1$ relationship, while the fraction of aquomethemoglobin remained constant. Further evidence that the high-spin species is high-spin hydroxymethemcglobin was provided by a study of the effect of $\mathrm{pH}$ on levels of high-spin form generated during methemoglobin reduction. A plot of the maximum value for the fraction of methemoglobin present as the high-spin form vs. pH (fig. 9) yielded a set of points that lie along a theoretical titration curve with a $\mathrm{p} K$ of 7.78. This is close to the $\mathrm{p} K_{\mathrm{a}}$ expected for the aquo-to-hydroxy transition of methemoglobin [18!.

During methemoglobin reduction, the absolute amount of high-spin hydroxy derivative is initially zero, increases to a maximum at approximately the midpoint of the reduction, and then declines to zero, paralleling the decrease in total methemoglobin. The fraction of total methemoglobin present as high-spin hydroxide derivative remains constant once the midpoint is reached. The amount of high-spin hydroxide varies with the conditions. At low ionic strength and in the absence of oxygen, the high-spin hydroxide reached a maximum of $10 \%$ at $50 \%$ reduction (fig. 10a). In the presence of $0.1 \mathrm{M} \mathrm{NaCl}$, a maximum of $12 \%$ is reached at $45 \%$ 

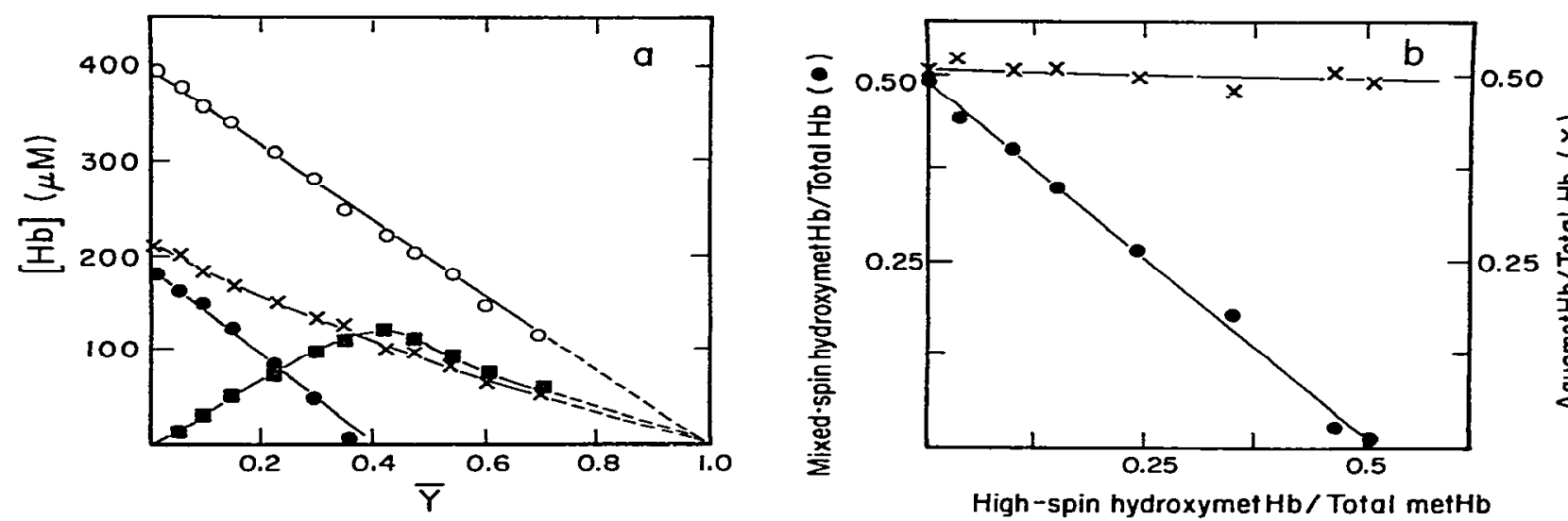

Fig. 8. Changes in the levels of the various species of ferric hemoglobin during the course of methemoglobin reduction. Reduction was carried out in the absence of oxygen at $\mathrm{pH} 7.8$ and $8^{\circ} \mathrm{C}$. Final concentrations; $400 \mu \mathrm{M}$ methemoglobin, $4 \mu \mathrm{M}$ cytochrome $b_{5}, 0.05$ unit cytochrome $b_{s}$ reductase $/ \mathrm{ml}$. I mM NADH. and $10 \mathrm{mM}$ dimethylglutaric acid (pH 7.8). In this multiple regression analysis. methemozlobin at pH 10.0 provided the spectrum of mixed-spin hydroxide methemoglobin, methemoglobin at pH $6.6 \mathrm{provided}$ the spectrum of aquomethemoglobin, and fluoromethemoglobin was used to approximate the spectrum of the high-spin, mixed-valence methemoglobin species. Values for each species were calculated as described in section 2.4 . $\bar{Y}$ is the fraction of hemoglobin subunits in the ferrous form. (a) Computed values for concentrations of the various species. Total ferric hemoglobin ( $O-O$ ), aquomethemoglobin $(x-x)$, mixed-spin hydroxymethemoglobin ( ), high-spin methemcoglobin ( $\mathrm{B}$ a). (b) The fraction of total methemoglobin present as the mixed-spin hydroxide species (- - ) and as the aquomethemoglobin species ( $x-x$ ) plotted vs. the fraction of total methemoglobin present as the high-spin species.

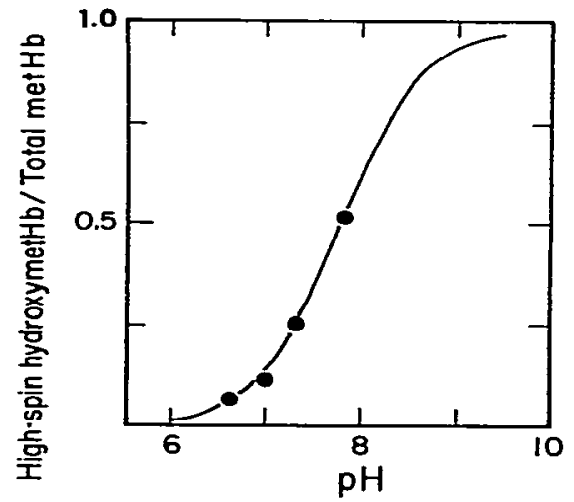

Fig. 9. Effect of $\mathrm{pH}$ on the maxiluum fraction of methemoglobin present as high-spin species during reduction. Conditions and procedures were the sarre as those described in fig. 8 , except for different $\mathrm{pH}$ values. The smooth curve is a titration curve for an acid with $\mathrm{p} K_{\mathrm{a}}=7.78$, calculated from the Henderson-Hasselbalch equation.

Fig. 10. Relationship between high-spin signal and valence state of hemoglobin. Reduction was carried out in the absence of oxygen as described in fig. $3 a . \bar{Y}$ is the fraction of hemoglobin subunits in the ferrous form. (a) No addition; (b) $0.1 \mathrm{M}$ $\mathrm{NaCl} ;$ (c) $50 \mu \mathrm{M}$ IHP, with and without $\mathrm{NaCl}$.

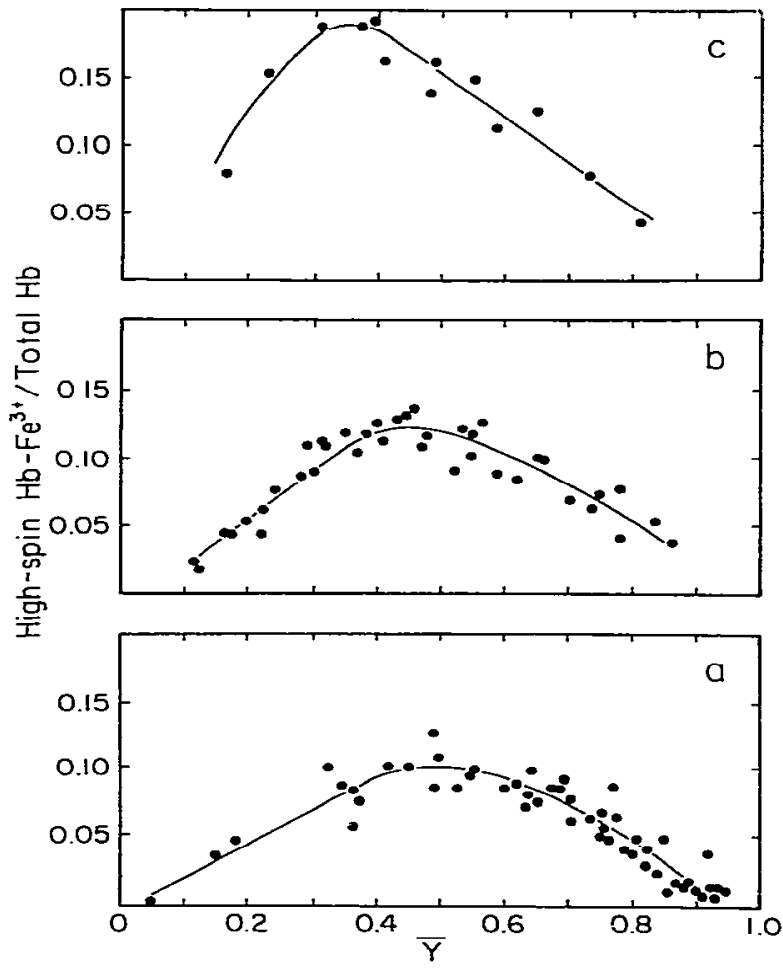


reduction (fig. 10b) and in the presence of IHP at either low or high ionic strength, a maximum of $19 \%$ is reached at $35 \%$ reduction (fig. 10c). In the presence of oxygen. high-spin hydroxide is detected only in the presence of IHP; under these conditions, a maximum concentration of $12 \%$ is seen at $30 \%$ reduction (data not shown).

\section{Discussion}

\subsection{MCD spectra of bovine hemeproteins}

The observed MCD spectra of the various oxidation and ligand states of bovine hemoglobin are very similar to those reported for human hemoglobin at this $\mathrm{pH}[5-7]$. The spectra of the oxidized and reduced forms of bovine cytocizome $b_{5}$ (fig. 1). however, differ in intensity in the visible region from those reported by Dolinger et al. [2] for rat liver cytochrome $b_{5}$ and by Vickery et al. [3] for pig liver cytochrome $b_{s}$. The Scret region does, however. agree with that obtained by Vickery et al. [1] for calf liver cytochrome $b_{5}$, for which the visible region was not reported.

\subsection{Reduction kinetics}

MCD proved to be an effective way to study the kinetics of methemoglejin reduction. It allowed us simultaneously to follow methemoglobin reduction and to monitor the level of ferrous cytochrome $b_{s}$. The intense signal of ferrous cytochrome $b_{5}$ allowed such studies to be carried out at low ratios of cytochrome $b$, to hemoglobin concentrations. Multiple regression analysis yielded concentrations of all the species present during the reduction in a reliable and self-consistent manner. The spectrum of each species, taken in the pure, isolated form, adequately represented that species when other forms were present, except in the case of cytochrome $b_{5}$.

The $\alpha$-peak of the spectrum of ferrous cytochrome $b_{5}$ showed a narrowing in the presence of forms of hemoglobin. At low ionic strength in the absence of oxygen, the narrowing was approx. 1 $\mathrm{nm}$ at the beginning of methemoglobin reduction and $0.5 \mathrm{~nm}$ at the completion of the reduction.
Under these conditions, the $K_{\mathrm{D}}$ changes by an order of magnitude (see fig. 5 a) which results in about (wice as much cytochrome $b_{5}$ complexed to hemoglobin at the beginning of the reduction than at the end. This is coincident with the 2 -fold change in the narrowing of the cytochrome $b_{5}$ signal. The significance of this finding has yet to be explored.

The results of section 3.2 demonstrate that the reduction of methemoglobin tetramer can be interpreted in terms of four apparent first-order rate constants. one for each of the four possible levels of oxidation of tetrameric methemoglobin. These four rate constants were derived in the context of a mc. Iel that assumed four sequential, nonreversible steps with each step being characterized by a first-order process. The model contains the expectation that the ratio $k_{4}: k_{3}: k_{2}: k_{1}$ should follow the pattern $4: 3: 2: 1$, which represents the number of available ferric subunits on each of the four possible tetrameric forms [16]. This expectation follows from the hypotheses that the intrinsic reaction rate of ferrous cytochrome $b_{5}$ with a ferric hemoglobin subunit is constant for each tetrameric species and that ferrous cytochrome $b_{s}$ interacts only with the ferric subunits of hemoglobin. The observed ratio pattern decreased more rapidly than the $4: 3: 2: 1$ pattern in the case of reduction under oxygenated conditions and therefore did not support the hypotheses. Reduction under aerobic conditions is an important test for the hypotheses because there should be no conformational changes in the protein to influence the rate. It is clear that the derived rate constants. while they can generate a curve that fits the data, are not easily interpretable in terms of the assumptions of the model. One is forced to postulate further that nonspecific changes occur as the tetramer passes through each step of the reduction which leads to decreases in the intrinsic ferrous cytochrome $b_{5}$-ferric subunit reaction rate. If such changes do occur, this model offers no insight into their origins.

In order to analyze the reduction kinetics from a more realistic approach we formulated the equilibrium state model II. This model postulates the formation of an intermediate, in the form of a protein-protein complex, and the ability of ferrous cytochrome $b_{S}$ to form nonproductive complexes 
with ferrous hemoglobin subunits. The equilibrium formulation (eq. 7) is based on Michaelis-Menten kinetics incorporating competitive inhibition, an approach used for enzymatic reactions where an enzyme-substrate complex is assumed. Although the electron transfer between cytochrome $b_{5}$ and hemoglobin is considered a nonenzymatic step, the separation of this reaction into complexation $\left(k_{i}^{\prime}\right)$ and then electron movement $\left(k_{t}^{\prime \prime \prime}\right)$ justifies the use of this kinetic approach.

Eq. 7 was able to describe satisfactorily the data for the reductions using the assumptions of a single $V_{\max }$ and with $K_{\mathrm{I}}$ equal to $K_{\mathrm{D}}$. In the presence of oxygen, a constant vaiue of $K_{\mathrm{D}}$ was obtained. This supports the reaction scheme of eq. 8 as well as the assumptions of the model. Although mechanisms cannot be definitively determined by kinetics analysis, this analysis is consistent with the following conclusions: (1) the rate constants of eq. 8 are independent of $i$; (2) ferrous and ferric hemoglobin subunits have the same affinity for cytochrome $b_{5}$; and (3) reaction is a two-step process with the rate of electron movement, $k^{\prime \prime \prime}$, being approximately equal to $0.1 \mathrm{~s}^{-1}$. In the absence of oxygen a dramatic increase in $K_{D}$ for the last two subunits in the reduction was observed. This implies that the ionic interaction between the two proteins is very sensitive to the R-to- $T$ conformational change of hemoglobin. Therefore, the equilibrium competitive model fits the data and yields parameters that are interpretable and consistent with known facts.

The data presented in sections 3.2 and 3.3 make it reasonable to assume that the structural transition, which occurs when two subunits of the tetramer are reduced, alters the structure of the two ferric subunits both externally and at the heme centers. The external changes in conformation result in iowered affinity for cytochrome $b_{s,}$, as represented by the increased $K_{\mathrm{D}}$. The change at the heme results in the complete conversion of the hydroxide methemoglobin component to a highspin form. Whereas the external change affects the rate of reduction, the change of spin state does not seem to alter the electron-transfer step in the reduction, since $V_{\max }$ is the same before and after this structural transition (fig. 4). Therefore, the ionic interaction between the two proteins is sensi- tive to tertiary structure and is the primary modulator of the reduction rate.

Results of reductions performed in the presence of IHP were difficult to interpret. In the presence of IHP and at $0.1 \mathrm{M}$ ionic strength under ariaerobic conditions, the transition point from low to high $K_{\mathrm{D}}$ occurred prior to 50\% reduction, but neither model I nor II was capable of satisfactorily fitting the data. In agreement with published data [19], the $\alpha$ - and $\beta$-subunits appear to show a marked difference in affinity for cytochrome $b_{5}$ in the presence of IHP, with the result that eight rate constants in model $I$ or eight terms in eq. 7 for model II are needed to describe th: $=$ reduction.

\subsection{High-spin hydroxymethemoglobin}

The high-spin methemoglobin that occurred in increased amount during reduction appeared to be a form of hydroxymethemoglobin. Hydroxymethemoglobin exists as a thermodynamic mixture of high- and low-spin states [20] both of which are represented in the MCD spectrum of hydroxymethemoglobin at pH 10.0 (fig. 1). The band at $575 \mathrm{~nm}$ is a characteristic low-spin band, while the negative deflection at $620 \mathrm{~nm}$ is part of a band centered near $600 \mathrm{~nm}$ representing the high-spin component. During the reduction of bovine methemoglobin the intensity of the low-spin band decreases and that of the high-spin band increases. When we analyzed the data, using hydroxymethemoglobir at pH 10 to represent the mixedspin species and fluoromethemoglobin to represent the pure high-spin hydroxymethemoglobin, the mixed-spin species was found to be replaced by the high-spin species in a $1: 1$ relationship during the reduction. This demonstrates that the spectra of fluoromethemoglobin, the high-spin component of mixed-spin hydroxymethemoglobin, and the high-spin species generated during the reduction, are all very similar and supports our hypothesis that the generated species is high-spin hydroxymethemoglobin. This hypothesis is further supported by the increase in absolute level of this species at high pH.

The occurrence of the maximum concentration of the high-spin component near the midpoint 0 i the reduction suggests that this component was the 
result of ferric subunits being driven to a higher spin state by structural changes induced by the ferrous subunits. The percent of ferric hemoglobin present as the high-spin hydroxide species reached a stable maximum beginning near the midpoint of the reduction (table 1 ). This indicates that a constant conformational 'pressure' was present during the second half of the reduction and is consistent with the transformation of the tetramer to the T-state. The effect of the allosteric effector, IHP, to increase the amount of high-spin hydroxide at earlier points in the reduction (fig. 3c) supports the above hypothesis since IHP can induce a T-like state in methemoglobin. The data support the model of Perutz et al. [21] that links spin state to structural conformation.

The existence of the high-spin hydroxide form as a component of methemoglobin has been discussed by Antonini and Brunori [14] and the expected absorption spectrum of pure high-spin hydroxymethemoglobin has been calculated by George et al. [22]. Near neutral pH, however. the predominant methemoglobin form is the high-spin aquo species with smaller contributions of low-spin aquo, low-spin hydroxide, and high-spin hydroxide forms. In partially reduced hemoglobin, Perutz [23] detected an increase in methemoglobin spin state and attributed it to an increase in the highspin hydroxide form. Moreover, Perutz et al. [18] have shown that $T$-state methemoglobin, induced by IHP, exhibited a hydroxide component of higher spin. The spectrum of this species was not obtained, however, because IHP does not bind at high $\mathrm{pH}$ where the hydroxide component predominates and methemoglobin thus remains in the R-state. In the current study, we establish an approximate MCD spectrum of this species (fig. 6) which resembles the spectrum of fluoromethemoglobin. The differences in the two spectra, which occur at the prominent charge-iransfer bands at 620 and $500 \mathrm{~nm}[24,25]$. may have arisen from the analytical procedures used to determine this spectrum. In fact, a closer similarity to the fluoromethemoglobin spectrum may be inferred from the excellent fit to the data (table 1 and fig. 8) generated by the use of fluoromethemoglobin as the putative high-spin hydroxide form. A similar agreement was calculated by George et al. [22] for the absorption band components of fluoro- and high-spin hydroxymethemoglobin. The MCD spectrum of high-spin hydroxymethemoglobin, being similar to that for the fluoride methemoglobin, thus verifies this calculated spectrum and indicates that the high-spin hydroxide species, like the fluoride derivative, exists completely in a spin state of $5 / 2$.

The derection of the high-spin hydroxide species was made possible because the mixed-valence state that is generated during anaerobic reduction induces a $T$-state conformation in bovine hemoglobin that results in the conversion of the hydroxymethemoglobin component to the completely high-spin state. The tetramer-dimer association constant for bovine her.oglobin $\left(4 \times 10^{6}\right.$ $\mathbf{M}^{-1}$ ) [15], which is an order of magnitude greater than that for human hemoglobin, may be responsible for this. In limited experiments we detected a smaller increase in high-spin hydroxymethemoglobin during the reduction of human methemoglobin. The subunit interactions that give rise to the different association constants are possibly responsible for the observed difference in the amount of high-spin hydroxymethemoglobin, making bovine hemoglobin an interesting protein for future work.

\section{Acknowledgements}

This work was supported in part by Grant AM-09250 from the United States Public Health Service, National Institutes of Health. The MCD spectropolarimeter was purchased with funds from a National Science Foundation equipment grant (PCM 76-82844). We gratefully acknowledge the kind assistance and expert advice of Dorothy $A$. Schafer and the fruitful discussions with Dr. Christopher Bull, Dr. Bo Hedlund, Dr. Jules Shafer and Dr. Russell Hille.

\section{References}

1 L. Vickery, T. Nozawa and K. Sauer, J. Am. Chrem. Soc. 98 (1976) 351 .

2 P.M. Dolinger, M. Kieiczewski, J.R. Trudell, G. Barth, R.E. 
Linder, E. Bunnenberg and C. Djerassi. Proc. Natl. Acad. Sci. U.S.A. 71 (1974) 399.

3 L. Vickery, A. Saimon and K. Sauer, Biochim. Biophys Acta 386 (1975) 87.

4 M. Kajiyoshi and F.K. Anan, J. Biochem. 81 (1977) 1327.

5 Y.A. Sharonow, A.P. Mineyev, M.A. Liushitz N.A. Sharonova, V.B. Zhurkin and Y.P. Lysov, Biophys. Struct. Mech. 4 (1978) 139.

6 J.I. Treu and J.J. Hoprield. J. Chem. Phys. 63 (1975) 613.

7 R.E. Linder, R. Records, G. Bart, E. Bunnenberg, $C$. Djerassi, B.E. Hedlund, A. Rosenberg. L. Seamans and A. Moscowitz. Biophys. Chem. 12 (1980) 143.

8 P.G. Righetti, G. Gacon, E. Gianazza, D. Lostanien and J.-C. Kaplan, Biochem. Biophys. Res. Commun. 85 (1978) 1575.

9 D.E. Hultquist, L.J. Sannes and D.A. Schafer, Prog. Clin. Biol. Res. 55 (1981) 291.

10 M.R. Mauk and A.G. Mauk, Biochemistry 21 (1982) 4730.

11 D.A. Schafer and D.E. Hultquist, Biochem. Biophys. Res. Commun. 95 (1980) 381.

12 L. Spatz and P. Strittnatter, J. Bîl. Chem. 248 (1973) 793.

$13 \mathrm{~J}$. Ozols, Biochemistry 13 (1974) 426.

14 E. Antonini and M. Brunori, Hemoglobin and myoglobin in their reactions with ligands, Frontiers of biology, vol. 21 (North-Holland, Amsterdam, 1971) p. 3.

15 S. Hanlon, G. Nagel, R. Boyd, R. Lewshenia, M. Forsherg and S. Cheung. Biochim. Biophys. Act.1 229 (1971) 359.

16 P.R. Bevington. Data reduction and error analysis for the physical sciences (McGraw-Hill, New York. 1969).

17 J.S. Olson, Methods Enzymol. 76 (1981) 652.

18 M.F. Perutz. J.K.M. Sandars. D.H. Chenery, R,W. Noble, R.R. Pennelly. L.W.M. Fung. C. Ho, 1. Giannini, D. Pörschke and H. Winkler, Biochemistry 17 (1978) 3640.

19 A. Tomodo, T. Yubisui, A. Tsuji and Y. Yoneyama, J. Bioi. Chem. 254 (1979) 3119.

20 M. Kotani, Adv. Quant. Chem. 4 (1968) 227.

21 M.F. Perutz. E.J. Heidner, J.E. Ladner, J.G. Beetlestone, $C$ Ho and E.F. SJade, Biochemistry 13 (1974) 2187.

22 P. George, J. Beetlestone and J.S. Griffith, in: Haematin enzymes (part I), eds. J.E. Falk. R. Lemberg and R.K. Morcon (Perganion Press, Oxford. 1961) p. 105.

23 M.F. Perutz. Nature 237 (1972) 495.

24 D.W. Smith and R.J.P. Williams. Struct. Bond. 7 (1970) 1.

25 T. Nozawa. S. Ookubo and M. Hatano. J. Inorg. Biochem. 12 (1980) 253. 